\title{
THE SPECIAL APPLICATIONS OF TEVATRON ELECTRON LENS IN COLLIDER OPERATION
}

\author{
X.Zhang, V.Shiltsev, FNAL, Batavia, IL 60510, USA \\ F.Zimmermann, CERN, Geneva, Switzerland \\ K.Bishofberger, UCLA, Los Angeles, CA 90095-1547
}

\section{Abstract}

Besides the Tevatron Electron Lens (TEL) runs as a R\&D project for Tevatron Beam-Beam Compensation [1], it is used daily as a Beam Abort Gap Cleaner for collider operations. It can also serve as beam exciter for beam dynamics measurements and as a slow proton or antiproton bunch remover. This report describes all these applications and related observations.

\section{TEL AS DC BEAM CLEANER}

\section{Beam and Abort Gap Loss}

Currently in the Tevatron, the protons are injected with a large longitudinal emittance, filling the RF bucket. And some of the DC beam coming from the Main Injector Coalescing Process is also injected. In addition, the protons slowly leak out of the buckets and migrate into the abort gap due to noise sources, which are not well understood [4]. The DC beam component usually reaches a level of about $200 \times 10^{9}$ during injection. These uncaptured protons are lost at the beginning of the ramp. At the end of a High Energy Physics (HEP) store, the DC beam amounts to $6 \times 10^{9}$ protons and antiprotons, which is enough to cause a quench in the Tevatron. When these numbers of protons are lost suddenly, their loss is evidenced by spikes in the CDF detector, which prevent the CDF from working properly. The detector may even be damaged. The Tevatron itself also quenches occasionally, if there are high losses at abort or on the ramp, due to the DC beam.

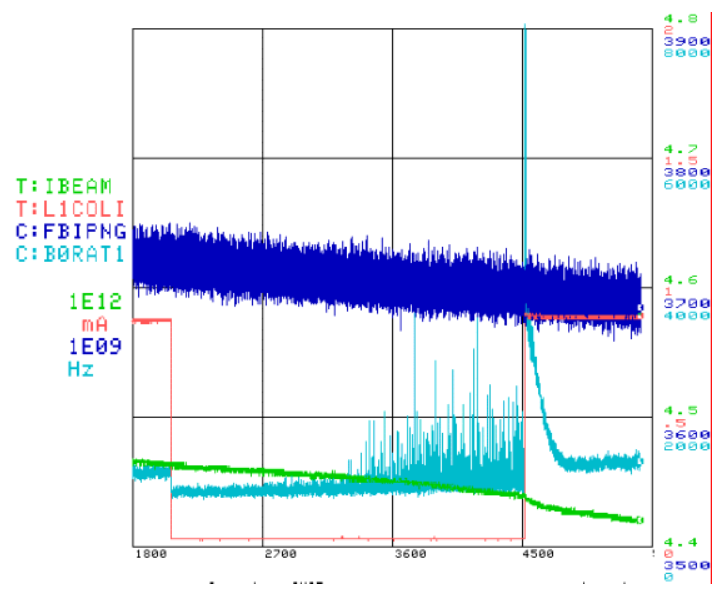

Figure 1: Beam intensity and loss during HEP store.

Figure 1 shows such a typical case for High Energy Physics Store. In this figure, the T:IBEAM is the total beam current in the Tevatron, C:FBIPNG is the total bunch current, the T:L1COLI is the average current of the electron beam and C:BORATI is the abort gap loss counter from the CDF detector. When the TEL was shut off (red trace), the abort gap loss started to grow after about 10min and the high spikes appeared.

\section{Beam Cleaning}

To clean out of the DC beam in the abort gap, the dipole beam-beam kick is used to excite the multipole resonance of particle oscillation to increase its oscillation amplitude, until the particles get lost at a limited aperture. Therefore, when the TEL was turned on, a significant amount of DC beam was lost, which is seen on T:IEBAM (green curve), while the bunched-beam lifetime $\mathrm{T}$ : FBIPNG did not change. A huge spike on the abort gap loss signal indicates that the DC beam in the abort gap was cleaned out by the TEL. Later the loss in the abort gap was stabilized without any loss spikes. By introducing the TEL for DC beam cleaning, we accelerate the loss of the DC beam and also distribute these losses over the warm section, which eliminates the big spikes in the detector, so that it is able to work properly, and we also reduce the quench likelihood during beam ramping and beam abort. Moreover, the slightly higher smooth baseline loss rate does not have any harmful effect. In DC beam cleaning operation, the electron beam of the TEL is placed about $2.5 \mathrm{~mm}$ beside the proton beam orbit horizontally and $1.5 \mathrm{~mm}$ below, as is shown in Figure 2 .

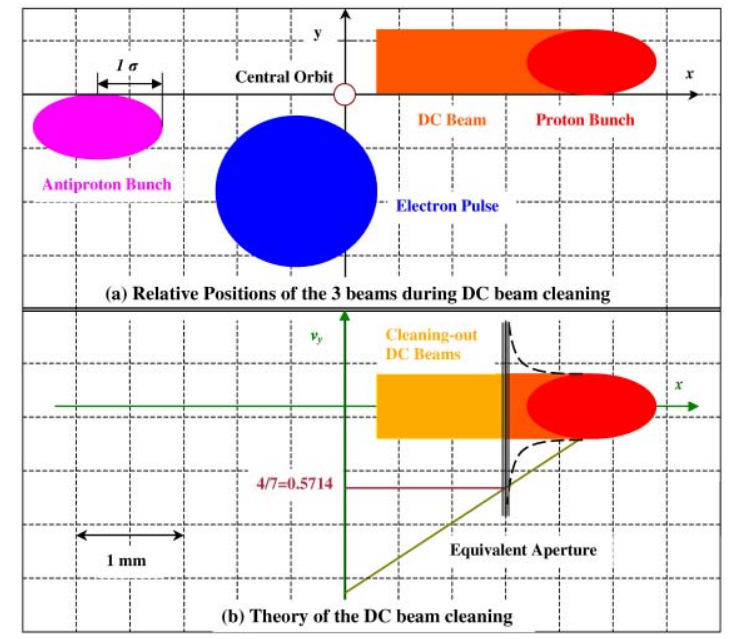

Figure 2: DC beam cleaning: (a) Beam postions (b) Physical mechanism of cleaning.

In Tevatron operation, we found that the most effective beam removal by TEL is to apply the electron beam kick of the TEL in every $7^{\text {th }}$ turn or $3^{\text {rd }}$ turn, which excites the $4 / 7^{\text {th }}$ order or $2 / 3^{\text {rd }}$ order resonance respectively. Figure 3 (below) shows a 1-turn snapshot taken when the TEL was 
operated in Abort Gap Cleaning State with 3 electron pulses pulsed in all 3 abort gaps on every $7^{\text {th }}$ turn. The blue trace is the electron gun cathode current. The pulse width is about $1 \mu \mathrm{s}$ and the peak amplitude is about $400 \mathrm{~mA}$. The green trace is the intensity signal of the TEL BPM, where the big upright spikes are the proton bunch signals (the proton pulse length is only 20ns). The downward pulses are electron pulses timed in the abort gap. The intensity of the antiproton bunches was 10 times less than that of the proton bunches. So they are the barely seen small downward spikes in between the proton bunches. The TEL excites the $7^{\text {th }}$ resonance of the protons in the abort gap and drives them out quickly.

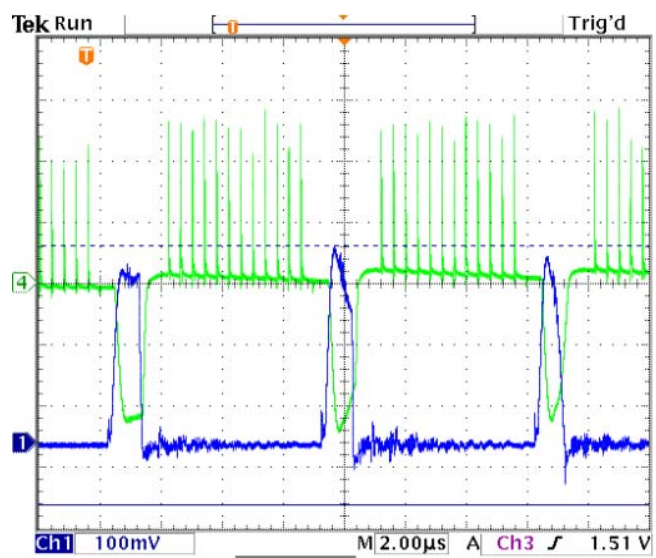

Figure 3: TEL for DC beam cleaning at 3-pulse every 7th turn.

\section{Mechanism of DC Beam Cleaning}

For normal Tevatron operation, the fractional part of the tune was chosen as 0.583 horizontally and 0.575 vertically. The most harmful resonance lines for the Tevatron are $4 / 7^{\text {th }}=0.5714$ and $2 / 3^{\text {rd }}=0.667$. When the particles in the DC beam lose energy by synchrotron radiation, their transverse oscillation amplitudes also increase, their tunes change, and, finally, they fall into the resonance band, where their oscillation amplitudes get larger and larger, until the particles hit the aperture and are lost. Usually it takes about $40 \mathrm{~min}$ for them to get lost with collimators open, while with the collimators closed at the beam and aperture restricted, it takes about $15 \mathrm{~min}$. However, with TEL excitation, this process takes only about 20sec. When the TEL is on, the tune of the DC beam particles is roughly described by:

$$
v=v_{0}+C_{V} \frac{x}{D_{x}}+\Delta v\left(x^{2}\right)+\Delta v_{T E L}
$$

where $v_{0}$ and $v$ are the tune of the on-momentum particles and DC-beam particles respectively; and $\Delta v_{T E L}$ the DCbeam particle tune change due to the electron beam. The tune also varies with the beam orbit[5], and $\Delta v\left(x^{2}\right)$ is the tune changes due to the particle orbit $x ; C v$ is the vertical chromaticity and $D_{x}$ the dispersion function at the TEL location which indicates the tune variation with particle momentum.
The DC beam cleaning mechanism is also shown in Figure 2(b). As the electron beam kicks the DC beam particles, their betatron oscillation amplitudes increase. The tune changes linearly with the particle position due to the chromaticity and quadratically due to high order field errors of the Tevatron. When the tune falls into the $4 / 7^{\text {th }}$ resonance, their oscillation amplitudes increase very fast until they hit the aperture and get lost. The equivalent aperture is the smallest aperture restricted by the collimation system transferred to the TEL location, which is about $3 \mathrm{~mm}$ from the helical beam orbit. Figure 4 shows the simulation results of the maximum particle oscillation amplitude for the linear lattice. The maximum amplitude is determined from the nonlinear force of the electron beam. Normally about $1 / 3$ of the DC beam is cleaned out of the abort gap, since the electron beam roughly occupies $1 / 3$ of the beam abort gap.

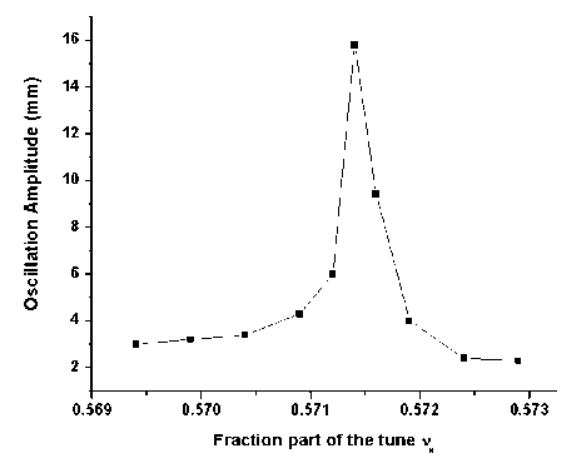

Figure 4: The oscillation amplitude of the particles near the $4 / 7$ th resonance line

\section{AS BEAM TICKLER}

The electron beam of the TEL can also be used as a beam 'tickler' for beam-dynamics measurements. To do so, we only need to modulate the electron beam current or position to produce the desired dipole kicks, and measure the beam response. Figure 5 (below) shows the method by which we modulated the electron current using white noise. The TEL was timed to specific pbar bunches to measure their tunes and emittance growth versus the noise strength.

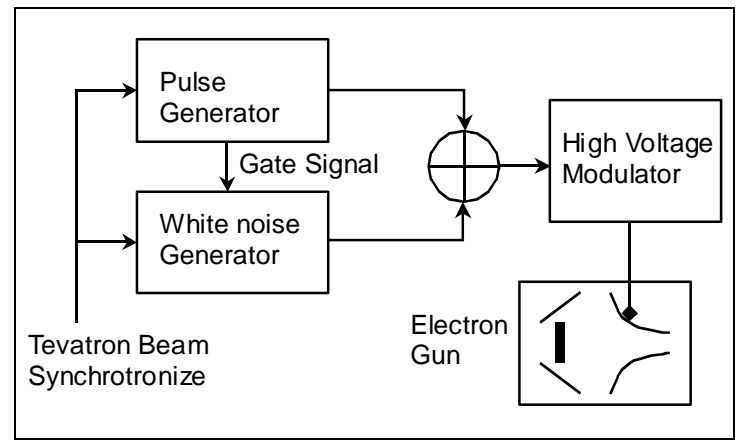

Figure 5: The scheme of TEL electron-current modulation. 
Figure 6 shows the waveform of electron current modulated by the white noise. The modulation depth was $70 \%$. This modulation was used to excite individual antiproton bunches for measuring bunch-to-bunch tunes.

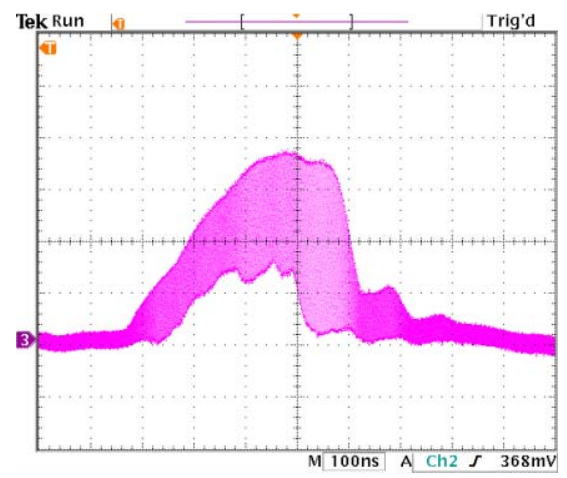

Figure 6: The waveform of the white noise modulated electron current.

The emittance growth due to the noise modulated electron beam kick was also observed [1]. It exhibits a quadratic dependence, which agrees with the theoretical prediction.

\section{Pbar Tune Measurement}

During the High Energy Physics store of Tevatron operation, normally the proton intensity is over ten times larger than the pbar intensity. The Schottky signal, which we are using now for tune measurement, is dominated by the proton signal. In order to detect the tune of the individual pbar bunches, we have to tickle the pbar beam.

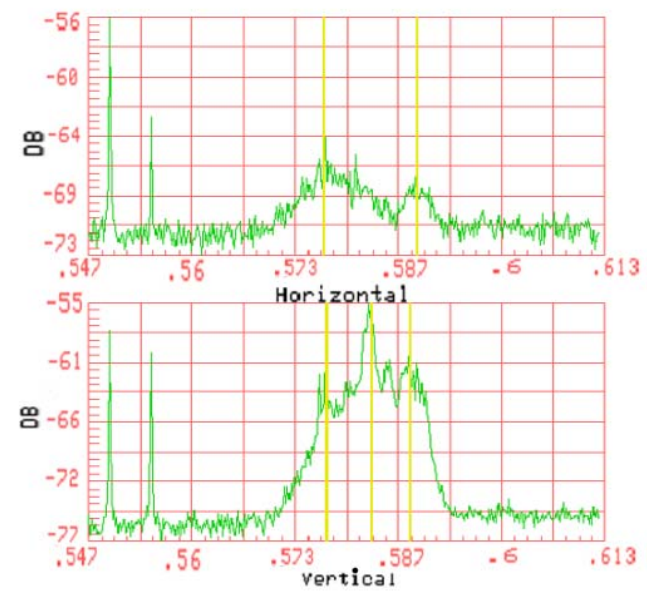

Figure 7: Tune signal from Schottky spectrum detector.

Figure 7 shows the Schottky spectrum of the beam without the TEL. It mainly shows the coalesced proton tune signal, which is about 0.575 in vertical and 0.59 in horizontal. By contrast, in Figure 8 the antiproton beam is excited by the TEL and we can infer the antiproton signal from the split peaks. For this measurement, we have controlled the modulation strength carefully in order not to blow up the pbar emittance too fast.

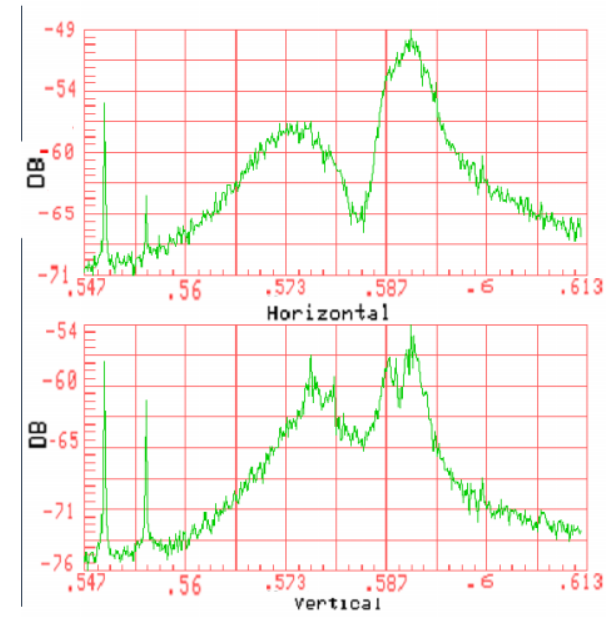

Figure 8: Tune signal with TEL excitation

\section{AS BEAM REMOVER}

The TEL acted as a controllable proton beam remover as shown in Figure 9. We can remove unwanted proton bunches one by one in a controlled way so that the loss in the Tevatron is kept below the dangerous level. During this operation, the electron beam is operated every $3^{\text {rd }}$ or $7^{\text {th }}$ turn to optimize the proton shaving speed.

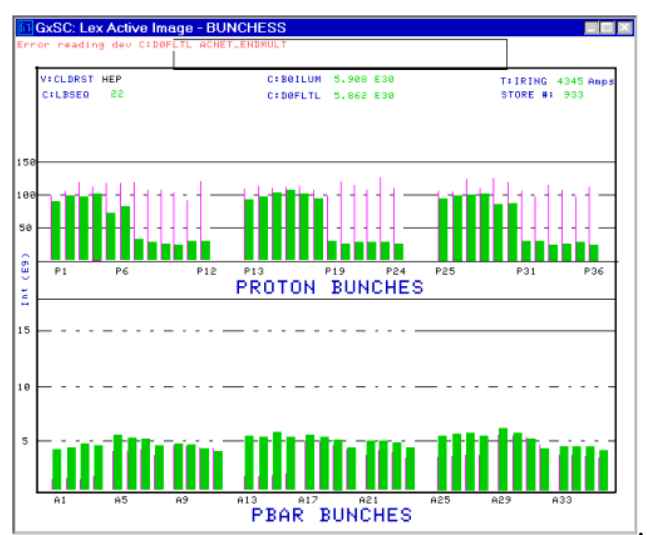

Figure 9: Tevatron display of bunch intensities during the removal of 18 proton bunches by the TEL.

\section{ACKNOWLEDGEMENT}

We thank the operations crew for their kind help and patience during our studies.

\section{REFERENCES}

[1] K. Bishofberger et al., this conference, TPPB083 and MOPA011

[2] X. Zhang et al., Beam Instrumentation Workshop 02, pp. $483-490$

[3] V. Shiltsev et al., Phys. Rev. ST Accel. Beams 2, 071001 (1999)

[4] Alvin Tollestrup et al., this conference, WPPB040

[5] Mike Martens et al., this conference, FPAB031 